\title{
Characterization of Barium Titanate Ceramic Powders by Raman Spectroscopy
}

\author{
Z. Lazarevićc ${ }^{a, *}$, N. Romčević ${ }^{a}$, M. Vijatović ${ }^{b}$, N. Paunović ${ }^{a}$, M. Romčević $a$, \\ B. Stojanović ${ }^{b}$ And Z. DohČević-Mitrović ${ }^{a}$ \\ ${ }^{a}$ Institute of Physics, Pregrevica 118, 11080 Belgrade, Serbia \\ ${ }^{b}$ The Institute for Multidisciplinary Research \\ Kneza Viseslava 1a, 11000 Belgrade, Serbia
}

Barium titanate, $\mathrm{BaTiO}_{3}$ ceramic powders were prepared by mechanochemical synthesis and by the Pechini method. A powder mixture of $\mathrm{BaO}$ and $\mathrm{TiO}_{2}$ was treated in a planetary ball mill in an air atmosphere for up to $1 \mathrm{~h}$, using zirconium oxide vial and zirconium oxide balls as the milling medium. After 60 min $\mathrm{BaTiO}_{3}$ phase was formed. In both ways $\mathrm{BaTiO}_{3}$ ceramics were sintered after $2 \mathrm{~h}$ on $1300^{\circ} \mathrm{C}$ without pre-calcinations step. The heating rate was $10^{\circ} \mathrm{C} \mathrm{min}^{-1}$. The formation of phase and crystal structure of $\mathrm{BaTiO}_{3}$ was approved by X-ray diffraction analysis and the Raman spectroscopy. The morphology and microstructure of obtained powders were examined by scanning electron microscopy method. Sharp phase transition from ferroelectric to paraelectric state was observed. The hysteresis loop is very well performed with regular sharp characteristic of ferroelectric materials.

PACS numbers: 77.84.-s, 81.07.Bc, 81.20.Ev, 83.85.Hf

\section{Introduction}

It is well known that barium titanate based materials provide properties that are important for a variety of electrical and electronical applications [1, 2]. Many researches have emphasized the importance of synthesizing process of $\mathrm{BaTiO}_{3}$ powder on the dielectric properties of the ceramic [3]. The microstructure control is, therefore, the key for enhancing the $\mathrm{BaTiO}_{3}$ ceramics electrical performances, and it is only possible by using non-conventional preparation methods such as sol-gel, oxalate, hydrothermal synthesis, citrates and polymeric precursors method, mainly based on the Pechini-type process [4]. The advantage of the Pechini method (polymeric precursor method) is based on the fact of its simplicity and possibility to hold the initial stoichiometry [5].

Beside, the mechanochemical method is characterized by the repeated welding, deformation and fracture of the constituent powder materials [6]. Under conditions of milling, it is found the releasing of heat, formation of new surfaces, formations of different crystal lattice defects and initiation of solid-state reaction. The accumulated deformation energy is the key of understanding the route of irreversible changes of crystal structure and consequently microstructure, causing in the change of properties of our material [7]. The objective of this work is to study the

* corresponding author; e-mail: lzorica@yahoo.com, lzorica@phy.bg.ac.yu feasibility of $\mathrm{BaTiO}_{3}$ formation and ceramics properties obtained from powders prepared by the polymeric precursors method (the Pechini process) and by mechanical activating of the constituent oxides.

A synthesis procedure and conditions for preparation $\mathrm{BaTiO}_{3}$ ceramic powders by the polymeric organometallic precursors method and by mechanochemical synthesis has been already described in previous paper [8]. The powders synthesized with both methods were pressed at $98.1 \mathrm{MPa}$, into $8 \times 2.5 \mathrm{~mm}^{2}$ pellets, using a cold isostatic press. The samples were sintered at $1300^{\circ} \mathrm{C}$ for $2 \mathrm{~h}$ (in the tube furnace "Lenton", UK). The heating rate was $10^{\circ} \mathrm{C} \mathrm{min}^{-1}$, with nature cooling in air atmosphere.

Characterization of the obtained samples was carried out by:

- By X-ray diffraction (XRD) analysis and scanning electron microscopy for barium titanate powders and for sintered samples were referred in more detail previously [8].

- Room temperature Raman spectra in spectral range from 100 to $800 \mathrm{~cm}^{-1}$, in backscattering geometry, were obtained by the micro-Raman analysis using Jobin Yvon T64000 spectrometer, equipped with nitrogen cooled charge-coupled-device detector. As excitation source we used the $514 \mathrm{~nm}$ line of an Ar-iron laser. The measurements were performed at $20 \mathrm{~mW}$ during $200 \mathrm{~s}$;

- The ferroelectrical properties of $\mathrm{BaTiO}_{3}$ ceramic samples were confirmed on the basis of the follow- 
ing characteristic parameter: coercive field, spontaneous and remnant polarization. Silver paste electrodes for electrical measurements were applied to the polished surfaces of $1 \mathrm{~mm}$ thick samples by the screen printing method. The silver paste was then polymerized at $600^{\circ} \mathrm{C}$ for $30 \mathrm{~min}$. The spontaneous $\left(P_{\mathrm{s}}\right)$ and remnant $\left(P_{\mathrm{r}}\right)$ polarization, as well as the coercive field $\left(E_{\mathrm{c}}\right)$, were determined by evaluating ferroelectric hysteresis loops obtained by means of a modified Sawyer-Tower circuit.

\section{Results and discussion}

The XRD results of powders (Fig. 1) indicate the formation of cubic phase of $\mathrm{BaTiO}_{3}$. The appearance of $\mathrm{X}$-ray reflections at $2 \theta=22.000,31.645,38.955,45.270$ and $56.135^{\circ}$ is in correlation with JCPDS (31-0174) standards. According to the previous studies [7], the structure of $\mathrm{BaTiO}_{3}$ may be cubic at room temperature. It can be observed that in the case of the Pechini process $\mathrm{BaTiO}_{3}$ powder is well crystallized but in the case of mechanochemistry process, significant amount of amorphous phase was detected [8]. The XRD results of sintered samples prepared by mechanochemical synthesis and by the Pechini process (Fig. 2) show the formation of tetragonal phase of $\mathrm{BaTiO}_{3}$, which is approved by the appearance of X-ray reflections at $2 \theta=$ $22.184,31.49,38.849,45.152,50.729,56.075$ and $65.711^{\circ}$ (JCPDS 05-0626). This could be confirmed using the Raman spectroscopy.

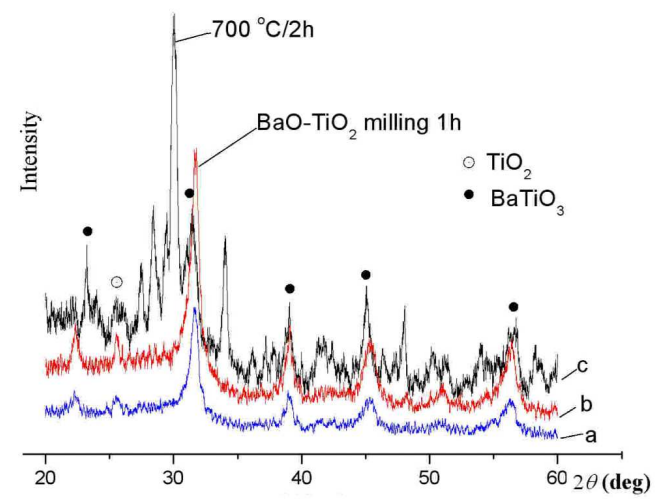

Fig. 1. X-ray diffraction patterns of the $(a)$ mixture $\mathrm{BaO}$ and $\mathrm{TiO}_{2}$ unmilled, (b) mixture $\mathrm{BaO}$ and $\mathrm{TiO}_{2}$ milled for $1 \mathrm{~h}$ (mechanochemically) and (c) $\mathrm{BaTiO}_{3}$ obtained after calcinations at $700^{\circ} \mathrm{C}$ for $3 \mathrm{~h}$ by the Pechini method.

The Raman spectra, for $\mathrm{BaTiO}_{3}$ ceramic powders samples obtained by the Pechini method and mechanochemical synthesis are presented in Fig. 3. $\mathrm{BaTiO}_{3}$ has five atoms and fifteen degrees of freedom per unit cell. In cubic phase it has $O_{h}$ symmetry, and the 15 degrees of freedom divided into the optical representations $3 F_{1 \mathrm{u}}+$ $F_{2 \mathrm{u}}$, while another $F_{1 \mathrm{u}}$ symmetry mode corresponds

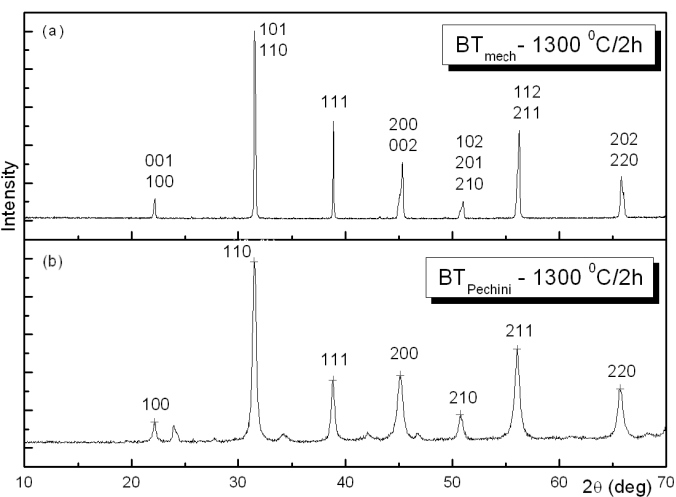

Fig. 2. X-ray diffraction $\mathrm{BaTiO}_{3}$ patterns obtained on sample sintered at $1300^{\circ} \mathrm{C}$ for $2 \mathrm{~h}$ and prepared by mechanochemical synthesis (a) and by Pechini method (b).

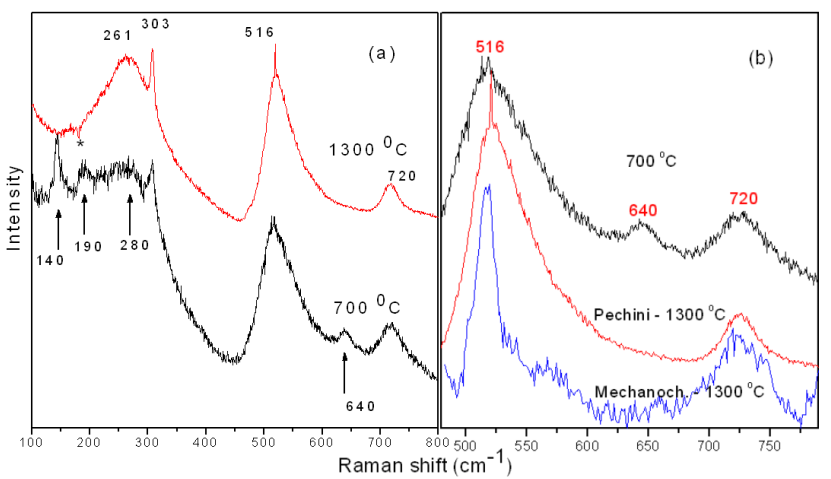

Fig. 3. (a) Raman spectra of $\mathrm{BaTiO}_{3}$ samples obtained by Pechini method, sintered at 700 and $1300^{\circ} \mathrm{C}$ and (b) compared with Raman spectra of the $\mathrm{BaTiO}_{3}$ sample produced by mechanochemical synthesis and sintered at $1300^{\circ} \mathrm{C}$.

to acoustical branch. At room temperature $\mathrm{BaTiO}_{3}$ is tetragonal and has $C_{4 v}$ symmetry. The frequency-covered range is from $100 \mathrm{~cm}^{-1}$ to $800 \mathrm{~cm}^{-1}$. Based on the crystallography, Raman-active modes for tetragonal $\mathrm{BaTiO}_{3}(P 4 m m)$ are $4 E(\mathrm{TO}+\mathrm{LO})+3 A_{1}(\mathrm{TO}+\mathrm{LO})+$ $B_{1}$ (TO $\left.+\mathrm{LO}\right)$, while no Raman-active mode is predicted for the cubic phase $(P m 3 m)$. The three $E(\mathrm{TO})$ modes with frequencies near to 190,280 and $516 \mathrm{~cm}^{-1}$ are labeled in Fig. 3a and b. As discussed above, the $190 \mathrm{~cm}^{-1}$ and the $516 \mathrm{~cm}^{-1}$ mode come from the $F_{1 \mathrm{u}}$ cubic phase modes, the $303 \mathrm{~cm}^{-1} E$ mode comes from the splitting of the cubic silent $F_{2 u}$ mode. The 140,303 , $640 \mathrm{~cm}^{-1}$, and the somewhat broader $720 \mathrm{~cm}^{-1}$ modes constitute the $E(\mathrm{LO})$ modes. The TO-LO splitting is fairly small and cannot be identified. The $A_{1}$ (TO) mode at $280 \mathrm{~cm}^{-1}$ is also shown in Fig. 3a. The intensity of the peak around $303 \mathrm{~cm}^{-1}$ was assigned to the overlap of $E(3 \mathrm{TO})+E(2 \mathrm{LO})+B_{1}$, which decreased with an increase in temperature and the mode disappeared above $T_{\mathrm{c}}$ where the structure became cubic. Many researchers are of the 
opinion that the Raman mode around $303 \mathrm{~cm}^{-1}$ is characteristic of the tetragonal $\mathrm{BaTiO}_{3}$. The Raman peak around $303 \mathrm{~cm}^{-1}$ shows a large decrease in intensity for the sample heat-treated at $700^{\circ} \mathrm{C}$ prepared by the Pechini method in comparison with that heat-treated at $1300^{\circ} \mathrm{C}$ prepared by mechanochemical synthesis suggesting that the tetragonal structure may be slightly sustained in the sample heat-treated at $700^{\circ} \mathrm{C}$.

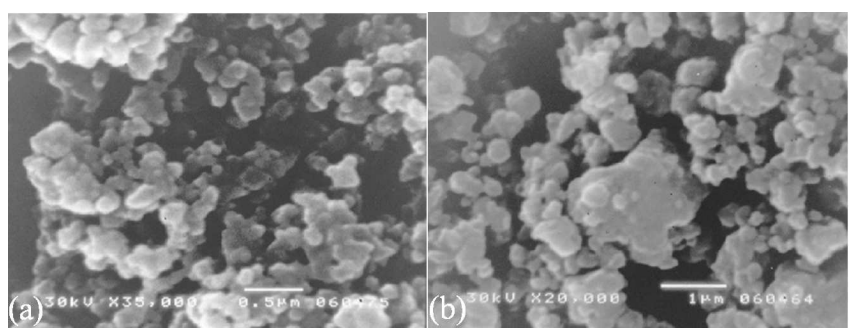

Fig. 4. The microstructure of $\mathrm{BaTiO}_{3}$ powders (a) synthesized by the Pechini process and (b) synthesized mechanochemically.

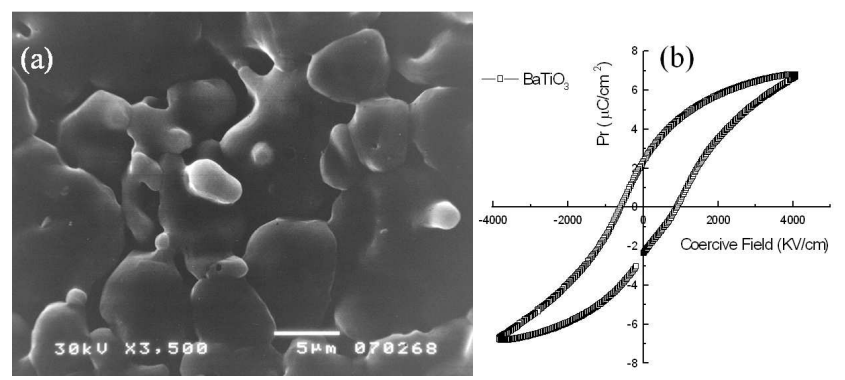

Fig. 5. (a) SEM image and (b) the hysteresis loop of the sample $\mathrm{BaTiO}_{3}$ synthesized mechanochemically and sintered at $1300^{\circ} \mathrm{C}$.

Figures 4 and 5a show the scanning electron microscopy (SEM) photographs of the $\mathrm{BaTiO}_{3}$ synthesized by the Pechini process and mechanochemically. The morphology of the powders consists of particles and its agglomerates. The agglomerates and particles depend on the synthesis method. The powder prepared mechanochemically processes higher number of agglomerates. The particles are bigger and with irregular shape. Average particle size of grains is about $100 \mathrm{~nm}$ and $250 \mathrm{~nm}$ for the Pechini and mechanochemical process, respectively.

It could be noticed that loop is very well performed with regular shape typical of ferroelectric materials (Fig. 5b). The remnant polarization was $2 \mu \mathrm{C} \mathrm{cm}-2$ and the coercive field was $1060 \mathrm{kV} \mathrm{cm}^{-2}$. The obtained values pointed to the regular microstructure of sintered specimens with small nanosized grains.

\section{Conclusion}

Barium titanate powder was prepared by two methods, polymeric organometallic precursors process and mechanochemically. In both ways of synthesis the formation of cubic phase is obtained. It can be observed that in the case of the Pechini process $\mathrm{BaTiO}_{3}$ powder is well crystallized but in the case of mechanochemistry process, significant amount of amorphous phase was detected. The sintered samples at $1300^{\circ} \mathrm{C}$ for $2 \mathrm{~h}$, shows the formation of tetragonal phase. The morphology of the powders consists of particles and its agglomerates, their dimensions depend on the synthesis method. The powder prepared mechanochemically possesses more agglomerates. The particles are bigger and with irregular shape. Average particle size is about $100 \mathrm{~nm}$ and $250 \mathrm{~nm}$ for the Pechini and mechanochemical process, respectively. The XRD and Raman measurements indicated formation of cubic structure $\mathrm{BaTiO}_{3}$ at lower temperature $\left(<700^{\circ} \mathrm{C}\right)$. However, the Raman spectrum suggested that tetragonal structure was achieved for sample $\mathrm{BaTiO}_{3}$ prepared by mechanochemical synthesis and cubic $\leftrightarrow$ tetragonal structure for sample $\mathrm{BaTiO}_{3}$ prepared by the Pechini method with calcinations step and sintered at $1300^{\circ} \mathrm{C} . \mathrm{BaTiO}_{3}$ sintered at $1300^{\circ} \mathrm{C}$ exhibit a hysteresis loop, confirming that the synthesized material possesses ferroelectric properties. From this research the formation of a pure $\mathrm{BaTiO}_{3}$ powder by both methods of synthesis is successfully approved and also the influence of used method on $\mathrm{BaTiO}_{3}$ properties is observed.

\section{Acknowledgments}

The authors gratefully acknowledge the Ministry of Science, Republic of Serbia for the financial support of this work (project No. 141028B).

\section{References}

[1] L.B. Kong, J. Ma, H. Huang, R.F. Zhang, W.X. Que, J. Alloys Comp. 337, 226 (2002).

[2] S. Ohara, A. Kondo, H. Shimoda, K. Sato, H. Abe, M. Naito, Mater. Lett. 62, 2957 (2008).

[3] W. Sun, J. Li, Mater. Lett. 60, 1599 (2006).

[4] P. Duran, F. Capel, J. Tartaj, D. Gutierrez, C. Moure, Solid State Ionics 141-142, 529 (2001).

[5] V. Vinothini, P. Singh, M. Balasubramanian, Ceram. Int. 32, 99 (2006).

[6] T. Tsuzuki, P.G. McCormick, J. Mater. Sci. 39, 5143 (2004).

[7] B.D. Stojanovic, J. Mater. Proc. Technol. 78, 143 (2003).

[8] Z.Ž. Lazarevic, M. Vijatovic, N.Ž. Romcevic, M.J. Romcevic, B. Stojanovic, in: Proc. Electroceramics IX Conf., Manchester 2008, electronic version by CD, C-023-P. 